\title{
Branched Polymers under Shear
}

\author{
Arash Nikoubashman ${ }^{* \dagger}$ and Christos N. Likos ${ }^{\dagger, *}$ \\ ${ }^{\dagger}$ Institute of Theoretical Physics, Heinrich Heine University of Düsseldorf, Universitätsstrasse 1, D-40225 \\ Düsseldorf, Germany and Faculty of Physics, University of Vienna, Sensengasse 8/12, A-1090 Vienna, Austria
}

Received October 6, 2009; Revised Manuscript Received December 16, 2009

\begin{abstract}
By employing a multiscale simulational approach that combines multiparticle-collision dynamics for the solvent with standard molecular dynamics for the monomers, we examine the behavior of dendritic macromolecules under shear. We analyze quantitatively the shape and anisotropy of the molecules and its dependence on the shear rate, the molecular generation, and the stiffness of the bonds. The role of hydrodynamics is brought forward by comparing our results with those obtained in the absence of coupling between monomers and solvent. We finally analyze the effects of charge and counterions in the system and complement our simulations with numerical results from Poisson-Boltzmann theory.
\end{abstract}

\section{Introduction}

The characteristics of rigid and soft mesoscopic particles in shear flow is of considerable interest from both the applied and the fundamental points of view. Colloidal suspensions under shear are omnipresent in our everyday life, for example, the flow of blood cells in our body or the painting of a wall with water-based paint. From a technological point of view, shear plays an essential role in a broad spectrum of applications of soft materials, such as drug delivery systems ${ }^{1}$ or as motor oil viscosity modifiers. ${ }^{2}$ The fundamental challenge lies therein, that typically soft matter systems consist of mesoscopically sized particles dissolved in a microscopic solvent, the characteristic time scales of the two being thus separated by many orders of magnitude. Any feasible computational approach must therefore be able to handle this vast discrepancy and manage to take into account the ubiquitous hydrodynamic coupling between the solvent and the solute.

Polymers and polymer assemblies exhibit a unique behavior in flow, which is related to their conformational degrees of freedom and distinguishes them sharply from rigid colloidal particles. Their flexibility leads to a simultaneous deformation of the coil and the fluid flow field, which strongly affect each other. Concomitantly, a rich dynamical behavior has been found for such deformable soft bodies, such as tumbling, tank-treading, rupture, stretching, and collapse. ${ }^{3,4}$ Thereby, many soft matter systems, ranging from rodlike colloids ${ }^{5}$ to star polymers, ${ }^{6,7}$ droplets, $^{8}$ capsules, and vesicles ${ }^{9}$ have been already investigated under shear flow conditions.

This work focuses on the conformations of dendritic molecules under shear. Although the equilibrium properties of dendrimers have already been analyzed extensively in the past two decades, ${ }^{10,11}$ the dynamic behavior of single dendrimers has not been studied thoroughly yet. These branched structures differ distinctively from other polymers, since the number of monomers grows exponentially with the generation number, while the volume of successive shells increases only as a power law of the generation number. Thus, the end monomers are folded back into the molecule, leading to a dense-core structure. ${ }^{12}$ This characteristic behavior has drawn some attention to the study of dendrimer melts, where both a deformation of the dendrimers and a shear thinning of the complex fluid have been observed theoretically ${ }^{13-15}$ as well as experimentally. ${ }^{16}$ \footnotetext{
de.

*Corresponding author. E-mail: arash.nikoubashman@uni-duesseldorf.
}

pubs.acs.org/Macromolecules

Published on Web 01/13/2010
Simulations of dendrimers or branched polymer under shear flow that take into account hydrodynamic interactions (HI) have been performed, employing the approximate Rotne-PragerYamakawa tensor; ${ }^{17-20}$ a microscopically based approach of the solvent dynamics and its coupling to the polymer degrees of freedom is however still lacking. To investigate the dynamics of flexible polymers in flow, we employ in this work a hybrid simulation approach, in which molecular dynamics simulations for the polymer are combined with a mesoscopic simulation technique for the solvent. This choice is due to the large length and time scale separation between the solvent molecules and the embedded polymer, which makes atomistic simulation studies prohibitively time-consuming. The employed particle-based simulation technique is called multiparticle-collision dynamics (MPCD) and has been introduced in 1999 by Malevanets and Kapral. ${ }^{21,22}$ Such simulations are especially valuable when analytical methods fail, as is the case for complicated molecules such as polymers, ultrasoft colloids, or semidilute solutions, where hydrodynamic interactions are screened only to a certain degree. Here we employ the MPCD technique for the solvent, coupled with molecular dynamics for the polymer, to examine the conformational properties of dendrimers under shear flow. We focus on the dependence of shape and anisotropy on the shear rate, the generation, the stiffness, and the presence of charge, and we critically compare the obtained results with one another and with previously found ones for linear chains and star-shaped polymers.

The rest of this paper is organized as follows: In section II we describe our dendrimer model. In section III, we briefly introduce the multiparticle-collision dynamics method. The results are then presented and discussed in section IV. There, we commence in section IV.A with the study of the relevance of both the rigidity and the generation number of the dendritic structures. Subsequently, we quantify the impact of hydrodynamic interactions in section IV.B, while the results for charged dendrimers are presented and discussed in section IV.C. Finally, we summarize the findings and draw our conclusions in section $\mathrm{V}$.

\section{Dendrimer Model}

A commonly used modeling approach for dendrimers is to treat the monomers (Kuhn segments) as bonded spherical beads, and to start with a central pair of joined monomers, the socalled zeroth generation $g=0$. A successive layer of monomers 
(generation $g+1$ ) is then formed by connecting two additional beads to each outer monomer of generation $g$. Thus, the functionality of the dendritic structure is $f=3$, and the number of monomers $n(g)$ taking part to a given generation $g$ follows a simple power law, i.e., $n(g)=2^{g+1}$.

In general, there are three different types of interactions between the individual monomers. The first contribution, $U_{\mathrm{mm}}(r)$, is a short-ranged, purely repulsive interaction given by a simple, shifted and truncated Lennard-Jones potential, which models the short-range, excluded-volume interactions between the monomers:

$$
U_{\mathrm{mm}}(r)= \begin{cases}4 \epsilon\left[\left(\frac{\sigma}{r}\right)^{12}-\left(\frac{\sigma}{r}\right)^{6}\right]+\epsilon & r \leq r_{\mathrm{cut}} \\ 0 & r>r_{\mathrm{cut}}\end{cases}
$$

with $r=\left|\mathbf{r}_{i}-\mathbf{r}_{j}\right|$ denoting the separation between the monomers $i$ and $j$, whose position vectors are $\mathbf{r}_{i}$ and $\mathbf{r}_{j}$, respectively. Equation 1 introduces an energy scale $\epsilon$ and a length scale $\sigma$, which will be taken as the units of energy and length, respectively, in what follows. In addition, the Boltzmann constant $k_{\mathrm{B}}$ and the absolute temperature $T$ will also be set to unity for the sake of simplicity. The cutoff distance has been chosen as $r_{\text {cut }}=2^{1 / 6} \sigma$, rendering the monomer-monomer interaction purely repulsive and thus suitable for an effective description of athermal solvents.

In addition to $U_{\mathrm{mm}}(r)$, bonded monomers within the same trifunctional unit interact via a FENE potential $U_{\mathrm{FENE}}(r)$, simulating the chemical bonds. ${ }^{23}$

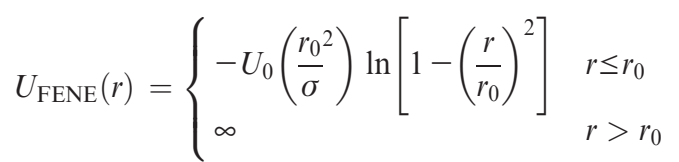

where the location of divergence $r_{0}$ determines the maximum bond length between two monomers and can be used along with $U_{0}$ to tune the stiffness of the polymer. To take into consideration the influence of the bond rigidity upon the dynamical behavior of dendrimers, we introduce two distinct pairs of values for $U_{\text {FENE }}(r)$. In this regard, we have chosen $U_{0}=10.0$ and $r_{0}=3.0$ for the first and $U_{0}=5.0$ and $r_{0}=4.0$ for the second set of parameters, the former representing stiffer and the latter softer bonds.

The last type of interaction to be included in our model is the Coulomb potential between charged monomers

$$
U_{\text {Coulomb }}=T \lambda_{\mathrm{B}} \frac{Z_{i} Z_{j}}{r}
$$

with charge numbers $Z_{i}$ and $Z_{j}$ and Bjerrum length $\lambda_{\mathrm{B}}$ given by

$$
\lambda_{\mathrm{B}}=\frac{e^{2}}{\varepsilon T}
$$

Here, the Bjerrum length has been chosen as $\lambda_{\mathrm{B}}=3.0 \sigma=7.2 \AA$ in order to mimic the characteristics of water with its relative permittivity $\varepsilon \approx 80$. Additionally, to guarantee charge neutrality of the whole system, the charged dendrimers have to be balanced by suitable populations of monovalent or multivalent counterions with valency $Z_{\mathrm{c}}$. Apart from the Coulomb interaction, these counterions also exhibit a short-ranged repulsion with each other and the monomers, described by eq 1 , which guarantees stability of the system against electrostatic collapse. ${ }^{24}$

The analysis of the shape and size of isolated dendrimers plays a key role in understanding not only the properties of the molecules themselves but also the thermodynamics of concentrated dendrimer solutions in a coarse-grained approach. ${ }^{25} \mathrm{~A}$ convenient measure for the overall size of polymers is given by the so-called radius of gyration $R_{\mathrm{g}}$, which is defined as

$$
R_{\mathrm{g}}{ }^{2}=\frac{1}{N}\left\langle\sum_{i=1}^{N}\left(\mathbf{r}_{i}-\mathbf{r}_{\mathrm{c}}\right)^{2}\right\rangle
$$

where $\mathbf{r}_{\mathrm{c}}$ denotes the center-of-mass position and $\langle\ldots\rangle$ denotes the statistical average over all conformations. To quantify the ordering of the individual monomer layers around the center of mass, it is convenient to consider the overall or generationdependent monomer density $\rho(r)$, which is defined as

$$
\rho(|\mathbf{r}|)=\left\langle\sum_{i=1}^{N} \delta\left(\mathbf{r}-\mathbf{r}_{\mathrm{c}}-\mathbf{r}_{i}\right)\right\rangle
$$

where $\delta(\mathbf{x})$ is the Dirac delta function.

\section{Simulation Method}

Simulation of pure fluids under planar shear has a long history. The so-called SLLOD simulation algorithm, developed in the mid-1980s, has long been the method of choice for the efficient simulation of pure solvents in Couette flow and the calculation of shear viscosities. ${ }^{26}$ In SLLOD, a velocity gradient tensor for the fluid is imposed from the outset, and Hamilton equations of motion of noncanonical form are solved numerically in the computer. ${ }^{27}$ When large molecules, such as polymer chains, are dissolved, the velocity profile of the solvent remains, however, unaffected, ${ }^{28}$ which implies that hydrodynamics is not taken properly into account.

To accurately take into account the hydrodynamic interactions mediated by the solvent, we employ a hybrid simulation approach, in which standard molecular dynamics (MD) simulations for the polymer are combined with the multiparticle-collision dynamics (MPCD) simulation technique. ${ }^{21,22}$ The latter is a mesoscopic, particle-based method consisting of alternating streaming and collision steps. During the streaming step the solvent particles propagate ballistically, so that the position of solvent particle $i$ at the next point of time $t+\Delta t$ is given by

$$
\mathbf{r}_{i}^{\prime}(t+\Delta t)=\mathbf{r}_{i}^{\prime}(t)+\Delta t \mathbf{v}_{i}^{\prime}(t)
$$

where $\mathbf{r}_{i}^{\prime}(t)$ denotes the position and $\mathbf{v}_{i}^{\prime}(t)$ the velocity of the $i$ th solvent particle at time $t$. In the collision step, the $N_{\mathrm{s}}$ solvent particles are first sorted into $N_{\text {cells }}=N_{x} \times N_{y} \times N_{z}$ cubic cells of edge length $a=\sigma$, each having a volume $V_{j}=a^{3}$. Subsequently, their velocities $\mathbf{v}_{i}^{\prime}$ are transformed via

$$
\mathbf{v}_{i}^{\prime}(t+\Delta t)=\mathbf{u}_{j}(t)+\Omega(\alpha)\left[\mathbf{v}_{i}^{\prime}(t)-\mathbf{u}_{j}(t)\right]
$$

with the center-of-mass velocity $\mathbf{u}_{j}$ of the corresponding cell and the norm-conserving rotation matrix $\Omega$ around a fixed angle $\alpha$.

A necessary prerequisite for any nonequilibrium simulation method is that it should reproduce the equilibrium results when the external driving fields (in our case, shear) are switched off. We have performed MPCD simulations with explicit solvent and dendrimers in equilibrium and calculated generation-resolved density profiles, comparing them with those arising from standard, equilibrium molecular dynamics employing the same dendrimer model. A typical density distribution is shown in Figure 1, demonstrating the excellent agreement between the two. It is evident that consecutive generations of monomers do not occupy concentric shells of their own as initially proposed by de Gennes and Hervet. ${ }^{29}$ Instead, a considerable probability of finding monomers of third or fourth generation in the area of the center is observable, supporting the validity of the dense-core model. ${ }^{12}$ 


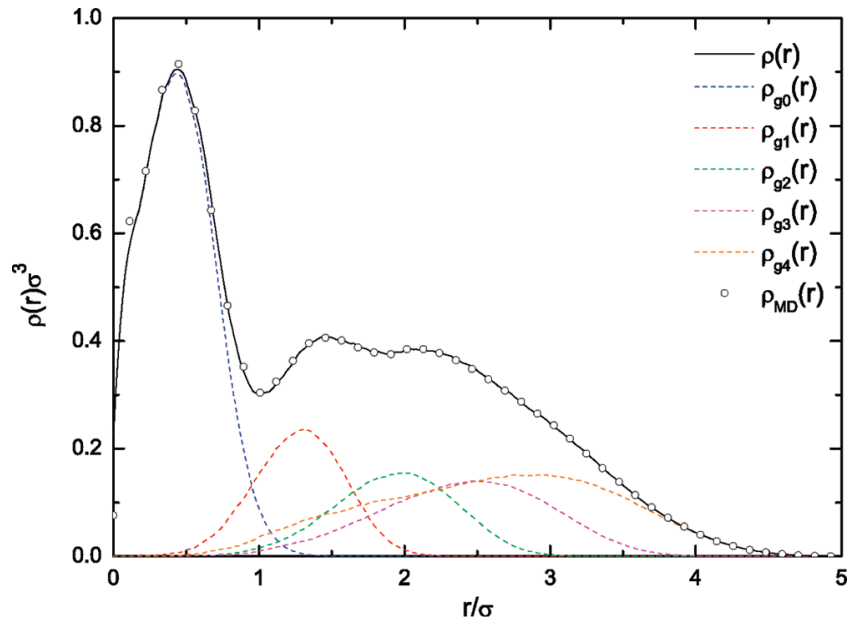

Figure 1. Generation-resolved monomer density distribution $\rho(r)$ of a trifunctional G4-dendrimer with $U_{0}=5.0$ and $r_{0}=4.0$. For the overall density profile, we show both the result from the MPCD (solid line) and that from a conventional MD without solvent (points).

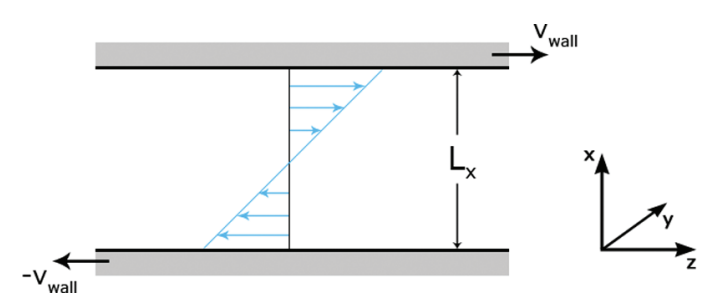

Figure 2. Schematic illustration of the simulation setup, demonstrating the shear $(z)$, gradient $(x)$, and vorticity $(y)$ directions.

The mean free path of a solvent particle is given by $\lambda=\Delta t \sqrt{ } T$, and it has been shown in ref 30 that Galilean invariance is violated for $\lambda<a / 2$. With the purpose of avoiding such a nonphysical behavior, all lattice cells $V_{j}$ are shifted by a randomly chosen vector drawn from the interval $[-\mathbf{a} / 2,+\mathbf{a} / 2]$ before each collision step. However, because of this shifting, partially occupied cells can emerge when the simulation box and cell boundaries do not coincide with each other. This procedure is unavoidable for small mean free paths $\lambda$, and it has been demonstrated in ref 31 that the boundary conditions have to be appropriately modified in such a case. We have tested the validity of our implementation by applying a Poiseuille flow as described in ref 32 and found that the solvent velocity correctly extrapolates to zero at the system boundaries. Shear flow with shear rate $\dot{\gamma}=v_{\text {wall }} / L_{x}$ is incorporated into the system by applying modified bounce-back boundary conditions, which involve the inversion of the particles' relative velocities to the walls instead of the absolute ones. We have favored this approach over other methods, e.g., Lee-Edwards boundary conditions, since the employed boundary conditions resemble more the actual experiment and lead to a spontaneous development of the desired linear velocity profile. Thus, the velocity profile is not externally imposed but is completely self-emerging. This feature offers corroboration of the validity of our approach. The geometry of this setup is schematically illustrated in Figure 2.

For the interaction between the solvent and solute particles, different strategies have been previously proposed. ${ }^{33,34}$ In the work at hand, the monomers are included in the MPCD collision step as point particles and thereby exchange momentum with the solvent. ${ }^{35}$ Moreover, no excluded-volume interactions between the solvent particles and the monomers are taken into consideration. This simplified approach is justified, since it has been shown in refs 36 and 37 that the desired long-range hydrodynamic interactions, which are theoretically described by the Zimm model for linear polymers, build up correctly.

Additionally, thermostatting is required in any nonequilibrium MPCD simulation whenever either isothermal conditions are required or viscous heating can occur. In the latter case, the viscous friction at the walls of the simulation box creates heat that results in a temperature rise. This increase in temperature then leads to a decrease of the local viscosity, which can have a tremendous influence on the velocity and density profile of the liquid. Basic requirements of any thermostat are that the conservation of the local momentum is not violated, the local flow profiles are not smeared out too much, and the global velocity distribution is not distorted notably. When there is homogeneous heating, the easiest way to maintain a constant temperature $T$ is to rescale the relative velocity components by a scale factor, which adjusts the total kinetic energy to the desired value. In our implementation of this scheme, we have used a local scale factor on the cellular level.

The monomer-monomer and (when present) monomercounterion dynamics is performed by using a usual MD algorithm (Verlet algorithm, $\Delta t_{\mathrm{MD}}=0.001 \sigma(\mathrm{m} / \epsilon)^{1 / 2}$ ). The simulation is carried out in a box of volume $V=L_{x} \times L_{y} \times L_{z}=20 \times 20 \times$ 100 with solvent particle density $\rho$ and at temperature $T=1$. In the case of charged dendrimers, we employ the Ewald summation algorithm $^{38,39}$ to properly take into account the long-range nature of the Coulomb interaction, expanding thereby the system to dimensions $V=50 \times 50 \times 100$ in order to minimize the impact of the charged periodic images. Furthermore, we have applied the aforementioned bounce-back boundary conditions in $x$-direction and periodic boundary conditions in the $y$ - and $z$-directions and employed the parameters $\alpha=130, \lambda=0.1$, and $\rho=5$ for the solvent dynamics. An important quantity of the solvent that can, among others, be calculated in this model is the dynamical viscosity $\eta$, which takes the form ${ }^{21}$

$$
\begin{gathered}
\eta=\frac{k_{\mathrm{B}} T \Delta t \rho}{a^{3}}\left(\frac{5 \rho}{(4-2 \cos \alpha-2 \cos (2 \alpha))(\rho-1)}-\frac{1}{2}\right) \\
+\frac{1-\cos \alpha}{18 a \Delta t}(\rho-1)
\end{gathered}
$$

The numerical value of $\eta$ in our simulations is $\eta=3.955(\epsilon m)^{1 / 2} /$ $\sigma^{2}(\approx 1 / 3 \mathrm{mPa} \cdot \mathrm{s})$.

\section{Results}

A. Neutral Dendrimers. The physical properties of polymers change considerably when an external flow field is applied. For small shear rates $\dot{\gamma}$, the conformation remains essentially unchanged compared to the equilibrium state. Only when $\dot{\gamma}$ exceeds a characteristic value, which corresponds to the longest relaxation time $\tau$ of the dissolved polymer in equilibrium, a structural anisotropy as well as an alignment is induced by the flow. For linear polymers ${ }^{40}$ as well as for the blob model of star polymers, ${ }^{6}$ analytic expressions for $\tau$ have already been derived in the Zimm model. However, since dendrimers are not fractal objects, an approach involving the blob model is not applicable, and hence a different solution is required. In this work, the dendrimers are treated in a similar fashion to linear polymers with an effective end-to-end length $L_{\text {eff }}=(2 G+1) b$. This assumption ignores the multitude of relaxation modes associated with the branching points, on the basis that they should be much faster and clearly separated by an overall, end-to-end relaxation mode that characterizes the dynamics of the molecule as a whole. As such, this approach is only justified in the case of very flexible dendrimers and low generation numbers. For higher generations or stiffer bonds, 


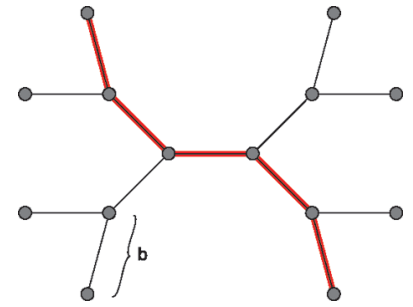

Figure 3. Schematic illustration of a trifunctional G2-dendrimer. The red path indicates the effective end-to-end length employed in this work to determine the Weissenberg number corresponding to a given shear rate.

dendrimers cannot be treated in this fashion anymore since their dense branching pattern induces more complex relaxation spectra, and there the molecule resembles a densely packed colloidal suspension in its interior. ${ }^{10}$

Figure 3 shows a schematic illustration of this approach, using the example of a trifunctional G2-dendrimer with bond length $b$, where the red path indicates the effective end-to-end length. Accordingly, the relaxation time $\tau$ reads as

$$
\tau=\frac{\eta b^{3}(2 G+1)^{3 \mu_{\mathrm{f}}}}{T}
$$

with the dynamic solvent viscosity $\eta$ and the Flory exponent $\mu_{\mathrm{f}}=0.588$.

Furthermore, to facilitate a comparison between dendrimers of different sizes, the so-called Weissenberg number $W i \equiv \dot{\gamma} \tau$ is introduced as a dimensionless measure of the shear rate. Evidently, the region $W i \geq 1.0$ is the most interesting, as we enter the nonlinear rheology regime of the dissolved macromolecule.

A convenient measure to quantify the structural properties and the alignment of polymers in flow is the average gyration tensor, $G_{\alpha \beta}$, defined as

$$
G_{\alpha \beta}(\dot{\gamma})=\frac{1}{N} \sum_{i=1}^{N}\left\langle r_{i, \alpha} r_{i, \beta}\right\rangle
$$

where $r_{i, \alpha}$ is the position of the $i$ th monomer relative to the center of mass and $\alpha, \beta \in\{x, y, z\}$ denote Cartesian components. This quantity is directly accessible in scattering experiments, and its diagonal components, $G_{\alpha \alpha}$, are the squared radii of gyration in $\alpha$ direction.

These diagonal components are shown in Figure 4 as functions of the Weissenberg number $W i$ for dendrimers of various stiffness and generations. First, it can be seen that despite the crude assumptions, the relaxation time $\tau$ of dendrimers defined in eq 10 is a surprisingly good description of the internal relaxation scale of dendrimers, since the individual curves show very similar behavior for molecules with different generations $G$. This fact holds especially true in the case of softer dendrimers with $U_{0}=5.0$ and $r_{0}=4.0$. Second, it is evident that, as expected, the extension of these dendritic structures increases with rising shear rate in the shear direction $(z)$, decreases in the gradient direction $(x)$, and is almost independent of $\dot{\gamma}$ in the vorticity direction $(y)$, A similar behavior has been also observed by Lyulin et al. ${ }^{17}$ and Bosko et al., ${ }^{19}$ who analyzed dilute dendrimer solutions under shear by means of Brownian dynamics simulations with a Rotne-Prager-Yamakawa interaction tensor for the hydrodynamic interactions. Moreover, the latter group has shown that this behavior is also visible for dendrimers in the melt under shear. ${ }^{13}$ However, these simulations lack a microscopically based approach of the solvent dynamics and its coupling to the polymer's degrees of freedom.
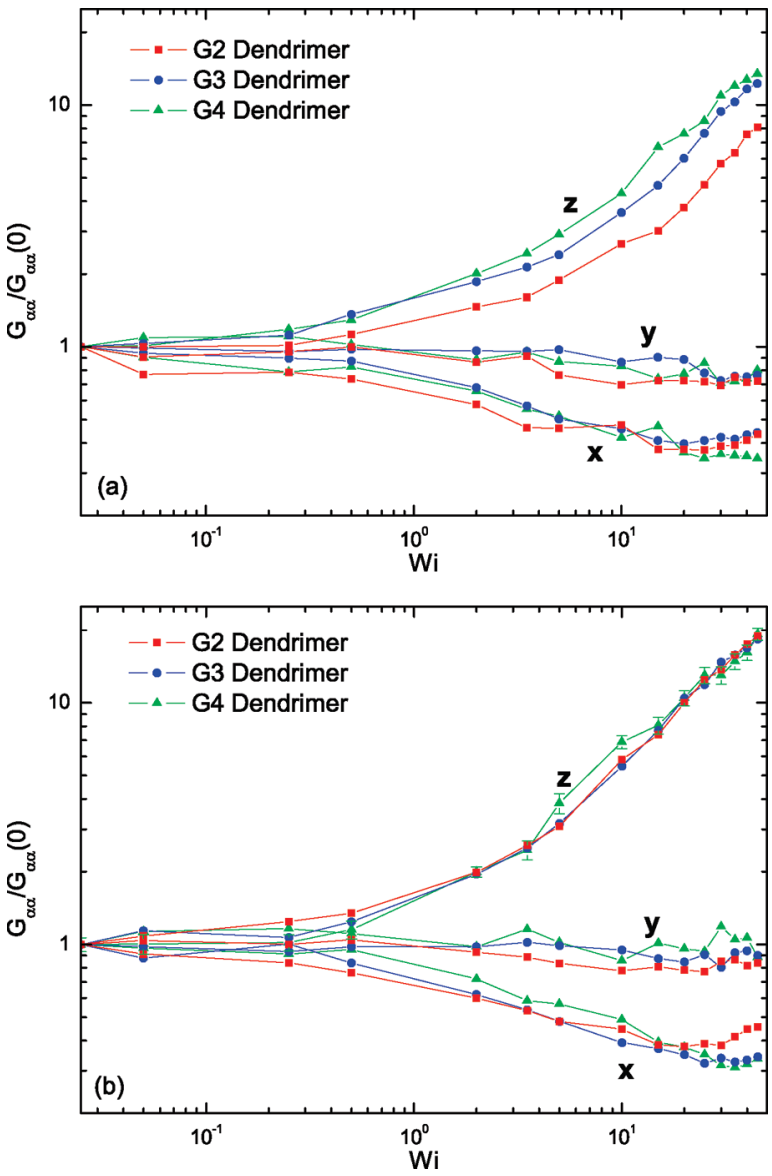

Figure 4. Normalized diagonal components $G_{\alpha \alpha}$ of the average gyration tensor as a function of the Weissenberg number $W i$ for G2-, G3-, and G4-dendrimers. (a) $U_{0}=10.0$ and $r_{0}=3.0$. (b) $U_{0}=5.0$ and $r_{0}=$ 4.0. Here and in subsequent figures we only show error bars in selected cases that represent also the rest, to avoid overcrowding of the plots.

With the purpose of analyzing the deviations from spherical symmetry in terms of the elongation in the shear direction, the normalized component $G_{z z}$ is plotted against $W i$ in Figure 5. A power law dependence is discerned in the regime $W i \gtrsim 1.0$. For rodlike colloids and linear polymers, such a behavior has already been observed and thoroughly analyzed, ${ }^{40}$ resulting in the establishment of a $W i^{2}$ power law. For small shear rates, star polymers exhibit a similar behavior, while a new scaling regime appears at higher shear rates, where the deformation scales linearly with the Weissenberg number. ${ }^{6}$ Dendrimers, on the other hand, show a completely different behavior, since neither a $W i^{2}$ dependence is visible for lower shear rates nor a proper linear growth is observable for larger values of $W i$. This is especially noticeable in the case of softer dendrimers with $U_{0}=$ 5.0 and $r_{0}=4.0$, where the exponent reads $\mu=0.861 \pm$ 0.022 . However, the finding of $\mu=0.921 \pm 0.120$ in the case of stiffer polymer bonds indicates that with increasing rigidity dendrimers resemble star polymers in this aspect of their behavior.

In Figure 6, the asphericity of dendrimers, characterized by the ratio $G_{1} / G_{3}-1 \geq 0$ between the largest and smallest eigenvalues of the average gyration tensor, is shown as a function of the Weissenberg number $W i$ for various generations $G$. In this regard, note that $G_{1} / G_{3}=1$ holds true for a rigid sphere, while this expression diverges in the limit of a long rod. In our case, both the rigidity and the size of the dendrimer play an important role. Referring to Figure 6a and comparing to the corresponding results in Figure 6b, we 

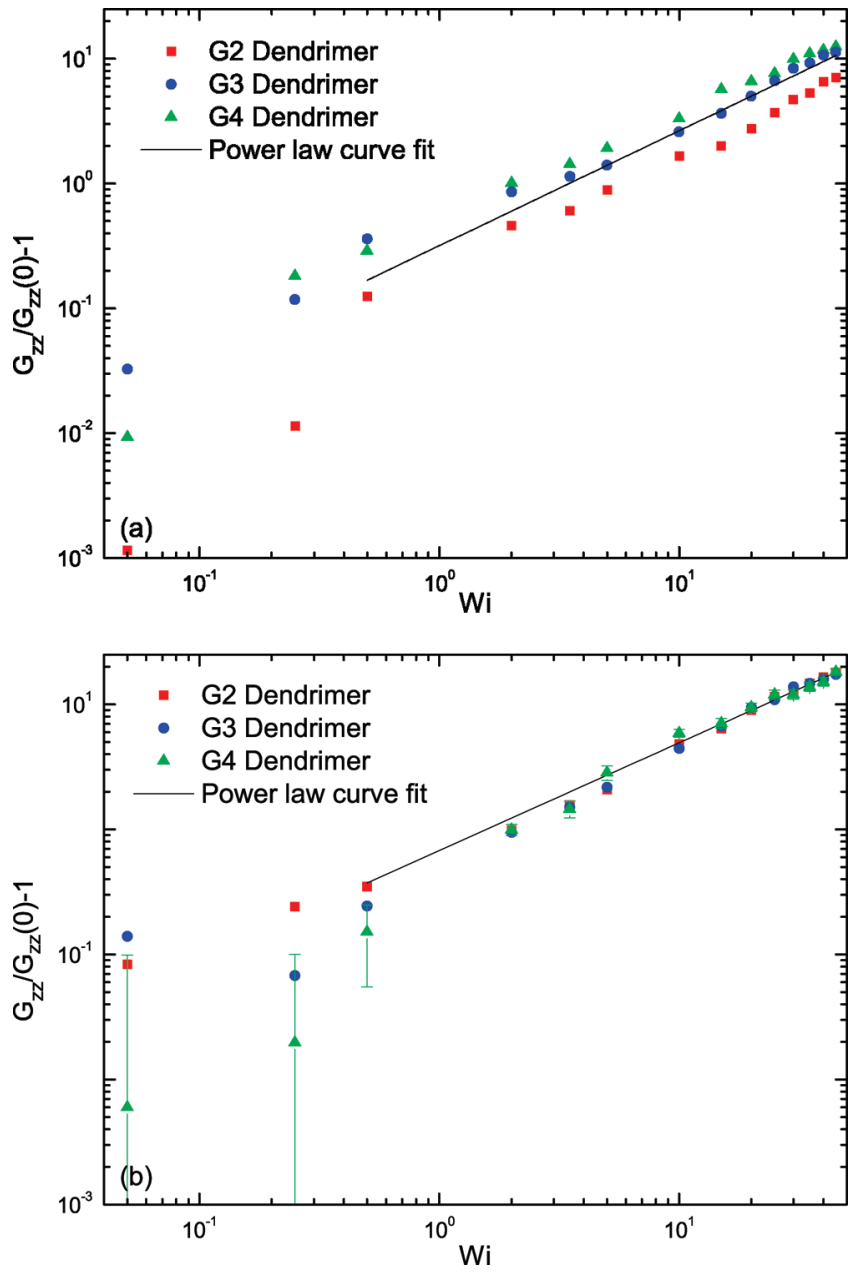

Figure 5. Normalized component $G_{z z}$ of the average gyration tensor as a function of the Weissenberg number $W i$ for G2-, G3-, and G4-dendrimers. (a) $U_{0}=10.0$ and $r_{0}=3.0$. (b) $U_{0}=5.0$ and $r_{0}=4.0$.

see that the anisotropy is stronger for the soft dendrimers in absolute terms, an intuitive result since these are more easily deformable than the stiff ones. Comparing among dendrimers of the same stiffness but different generations, we notice that the asphericity grows with the generation number. This appears counterintuitive at first, since it is known that, in equilibrium, sphericity is restored as the maximum generation number grows. ${ }^{10}$ However, proper understanding of the trends reported here requires consideration of the fact that dendrimers of larger generation are also more extended in space than their low-generation counterparts. As such, they are also exposed to a stronger influence from the streaming solvent, whose (undistorted) velocity profile grows linearly with the distance from the center of the box in the hereby simulated Couette flow. Concomitantly, large generation dendrimers are influenced more strongly, and a net larger asphericity results.

The resistance of a macromolecule against alignment with the flow direction in the presence of shear can be described by the orientational resistance parameter $m_{G}(W i)$. This quantity is related to the angle $\chi_{G}$, which lies between the eigenvector with the largest eigenvalue of the gyration tensor $G_{\alpha \beta}$ and the flow direction. For the chosen geometry, symmetry considerations imply that all off-diagonal elements of the symmetric tensor $G_{\alpha \beta}$ for which one index is given by the vorticity direction $y$ vanish. Accordingly, the eigenvectors associated with the largest and smallest eigenvalues lie in the $(x, z)$-plane, and a straightforward calculation yields for the orientational
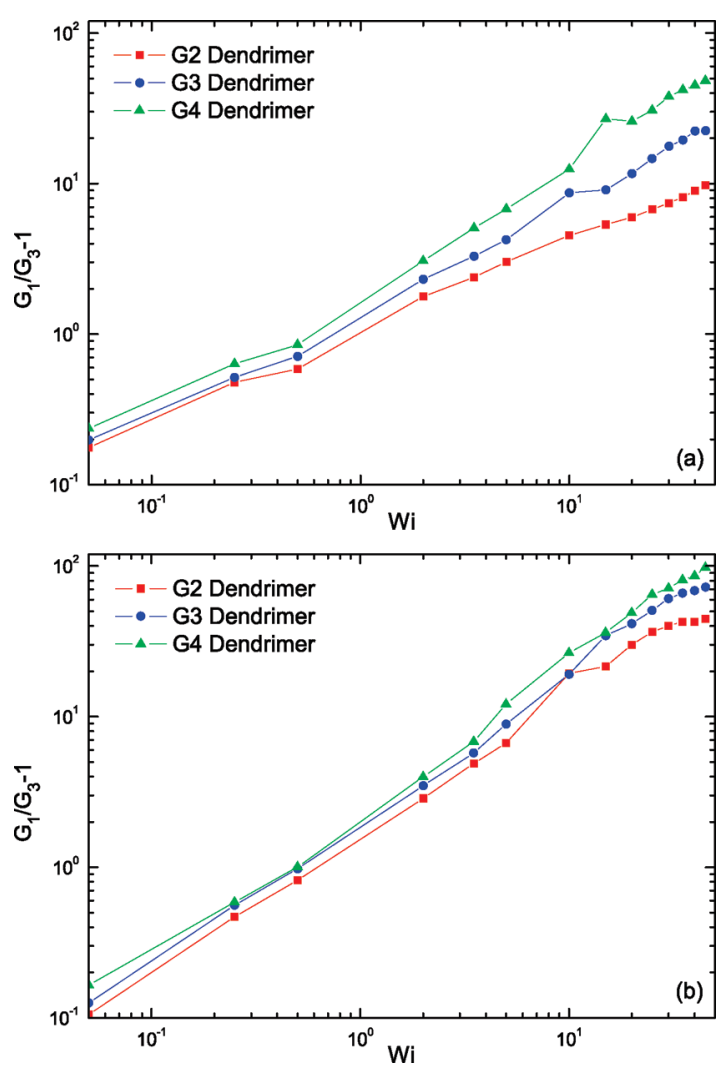

Figure 6. Ratio of the largest $\left(G_{1}\right)$ and smallest $\left(G_{3}\right)$ eigenvalues of the average gyration tensor as a function of the Weissenberg number $W i$ for G2-, G3-, and G4-dendrimers. (a) $U_{0}=10.0$ and $r_{0}=3.0$. (b) $U_{0}=5.0$ and $r_{0}=4.0$.

resistance:

$$
\frac{m_{G}}{W i} \equiv \tan \left(2 \chi_{G}\right)=\frac{2 G_{x z}}{G_{z z}-G_{x x}}
$$

For several systems, including rodlike colloids and linear polymers, it has been shown that at low Weissenberg numbers the scaling relations $G_{x z} \sim \dot{\gamma}$ and $\left(G_{z z}-G_{x x}\right) \sim \dot{\gamma}^{2}$ hold true, ${ }^{40,41}$ implying that $m_{G}$ is independent of $W i$. Results from this work for various trifunctional dendrimers are depicted in Figure 7. Unlike the aforementioned cases, the results do not approach a plateau for small shear rates. However, it is well visible that the data for different generations $G$ collapse onto an universal curve for larger shear rates and that the orientational resistance can be described by a power law $m_{G} \sim W i^{u}$, with $\mu=0.488 \pm 0.037$. This value is slightly smaller than the exponent for self-avoiding linear polymers, which was determined as $\mu=0.54 \pm 0.03$ in refs 42 and 43 . For star polymers, an even larger exponent $\mu=$ $0.65 \pm 0.05$ has been found in ref 6 . Thus, dendrimers exhibit the smallest orientational resistance, in comparison with the aforementioned common polymeric systems.

B. Impact of Hydrodynamics. Hydrodynamic interactions play a key role in many physical systems and processes. Hence, it is very important to study and quantify their relevance. To analyze the impact of hydrodynamics qualitatively, it is pertinent to study the deviations of the fluid flow from the linear profile due to the presence of a dendrimer. Figure 8 shows the fluid streamlines in the flow-gradient plane in the presence of a G4-dendrimer, which is placed at the center of the box. Evidently, the fluid flow in the vicinity of a dendrimer differs distinctively from that of a rigid, spherical body, since the solvent penetrates into the area 

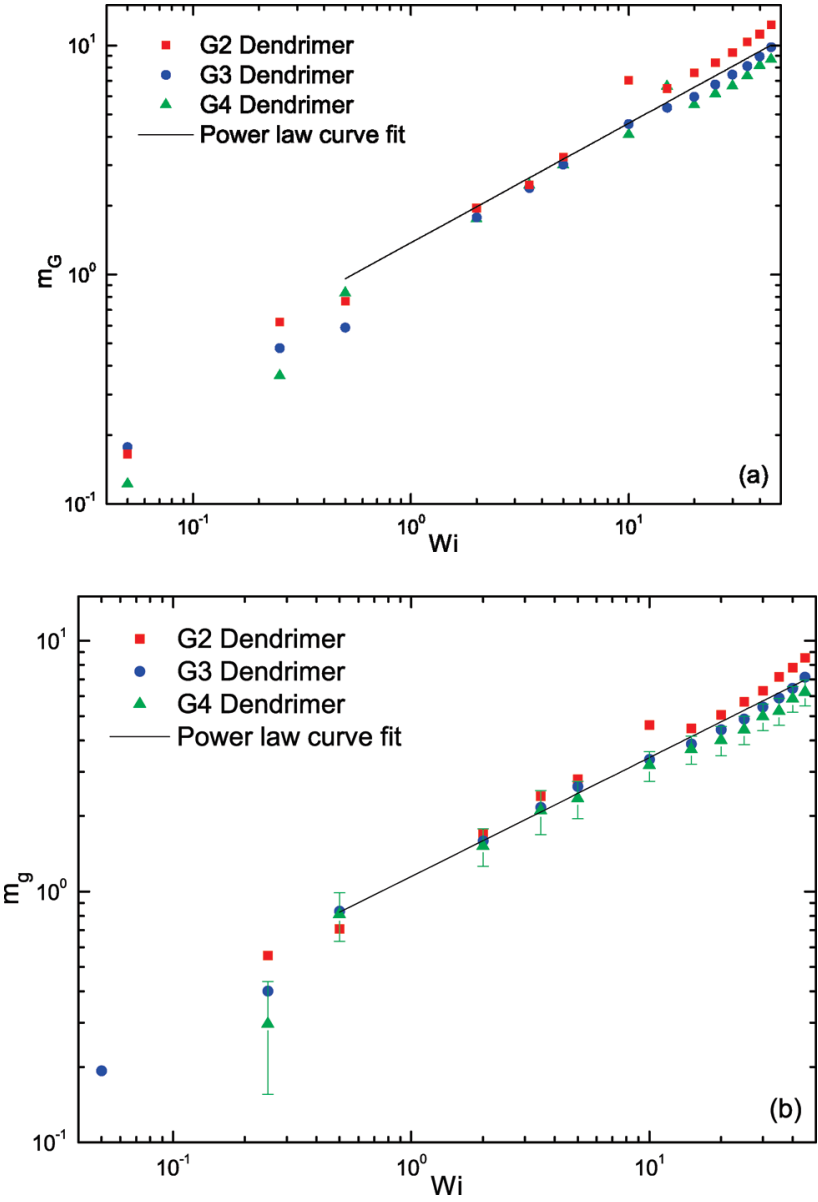

Figure 7. Orientational resistance $m_{G}$ as a function of the Weissenberg number $W i$ for G2-, G3-, and G4-dendrimers. (a) $U_{0}=10.0$ and $r_{0}=$ 3.0. (b) $U_{0}=5.0$ and $r_{0}=4.0$.

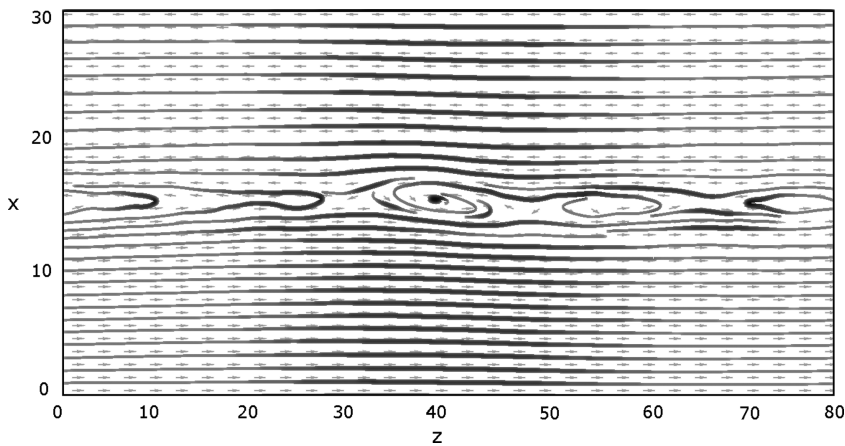

Figure 8. Fluid stream in the flow-gradient plane for a G4-dendrimer sheared with $W i=10$.

occupied by the polymer. Here it is well visible that in the region where the fluid coexists with the dendrimer the externally imposed flow field is strongly screened and the fluid velocity is no longer aligned with the shear flow direction, but rather rotates around the polymer's center of mass. Outside the region covered by the dendritic structure, the fluid adapts to the central rotation by generating both a counter-rotating vortex and consequently two hyperbolic stagnation points of vanishing fluid velocity. However, the fluid in the corona of the dendrimer follows the externally imposed flow to a considerable extent.

More quantitative results are presented in Figure 9, where the deviation of the average fluid velocity from the undisturbed linear profile is shown for different shear rates $\dot{\gamma}$ and
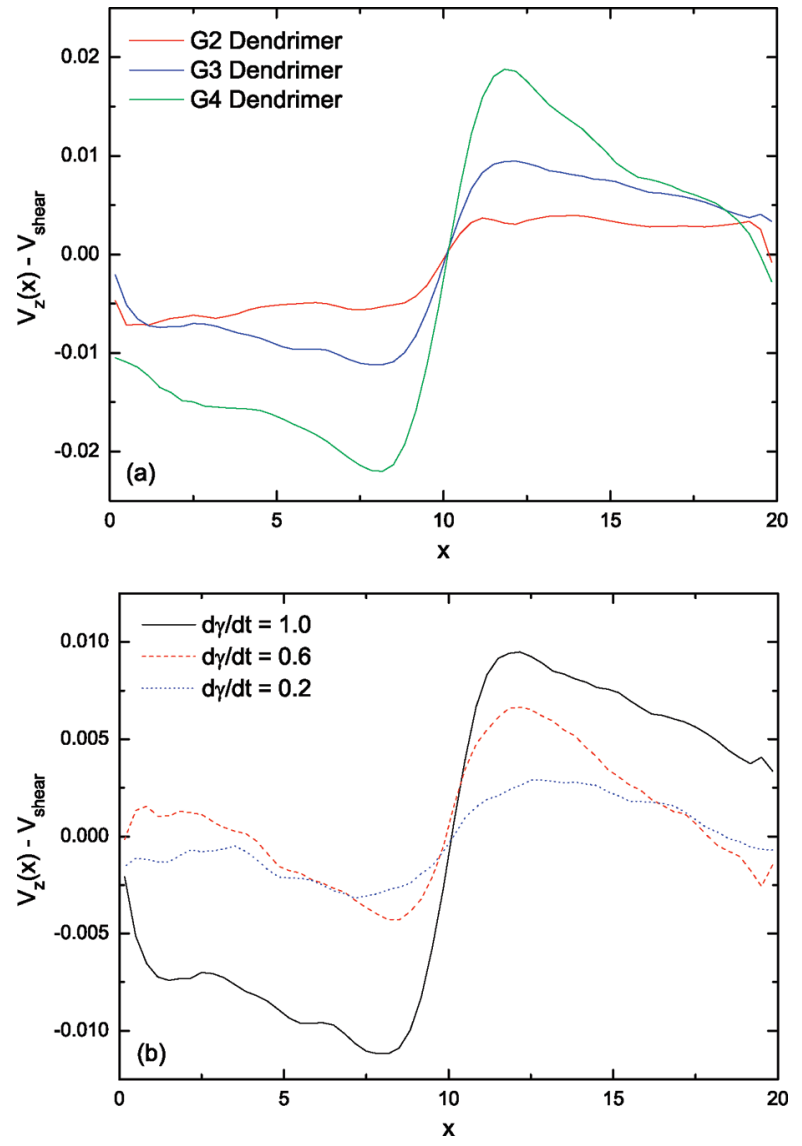

Figure 9. Deviation of the fluid flow from the linear profile due to the presence of a dendrimer. The simulation was carried out for a system of size $V=20 \times 20 \times 100, \lambda=0.1$ and $\rho=5$. Furthermore, the presented velocity profiles have been recorded at the center of the simulation box. (a) Impact of G2-, G3-, and G4-dendrimers on the velocity flow field at a shear rate $\dot{\gamma}=1.0$. (b) Influence of different shear rates on the degree of hydrodynamic interactions with a G3-dendrimer.

generations $G$, indicating that the deviation distinctively increases for larger $\dot{\gamma}$ and $G$. It is also apparent that the results are distributed point-symmetrically around the dendrimer's center of mass and do not converge to zero at the boundaries due to the long-ranged nature of hydrodynamic interactions. Furthermore, these plots show clearly that the dendrimers counteract the imposed flow field, since the deviations have an opposite sign to the velocity profile.

The hydrodynamic interactions manifest themselves also on the conformations of the flexible, dissolved objects. To quantify these, the conformational characteristics of a G4dendrimer with $U_{0}=5.0$ and $r_{0}=4.0$ have been analyzed in a separate simulation without hydrodynamics. Such conditions can easily be achieved via Brownian dynamics, where the Oseen tensor is set to the identity matrix $\mathbf{H}_{i j}=\mathbf{I}$, and the velocity field of the Couette flow is superimposed:

$$
\begin{aligned}
\mathbf{r}_{i}(t+\Delta t)=\mathbf{r}_{i}(t) & +c_{1} \Delta t \mathbf{v}_{i}(t)+2 \Delta t \dot{\gamma}\left(r_{i, x}-\frac{L_{x}}{2}\right) \hat{\mathbf{z}} \\
& +c_{2} \Delta t^{2} \mathbf{a}_{i}(t)+\mathbf{r}_{\mathrm{G}}
\end{aligned}
$$

In eq $13, r_{i, x}$ is the position of the $i$ th bead in gradient direction and $\hat{\mathbf{z}}$ denotes the unit vector in shear direction. The coefficients read $c_{1}=(1-\exp (-\xi \Delta t)) /(\xi \Delta t)$ and $c_{2}=$ $\left(1-c_{1}\right) /(\xi \Delta t)$ with friction coefficient $\xi$, while $\mathbf{a}_{i}$ denotes the acceleration and $\mathbf{r}_{\mathrm{G}}$ a random variable drawn from a bivariate Gaussian distribution with zero mean value and variance 


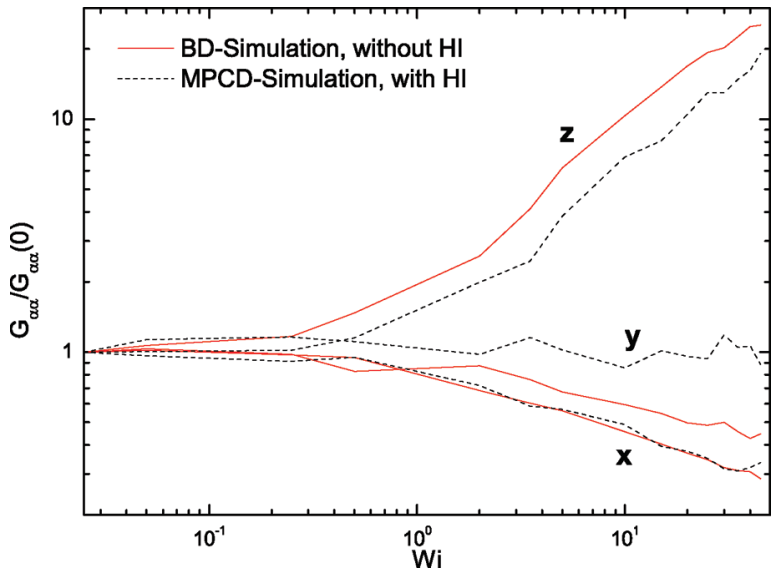

Figure 10. Normalized diagonal components $G_{\alpha \alpha}$ of the average gyration tensor as a function of $W i$, comparing between a simulation without (BD) and with (MPCD) hydrodynamic interactions.

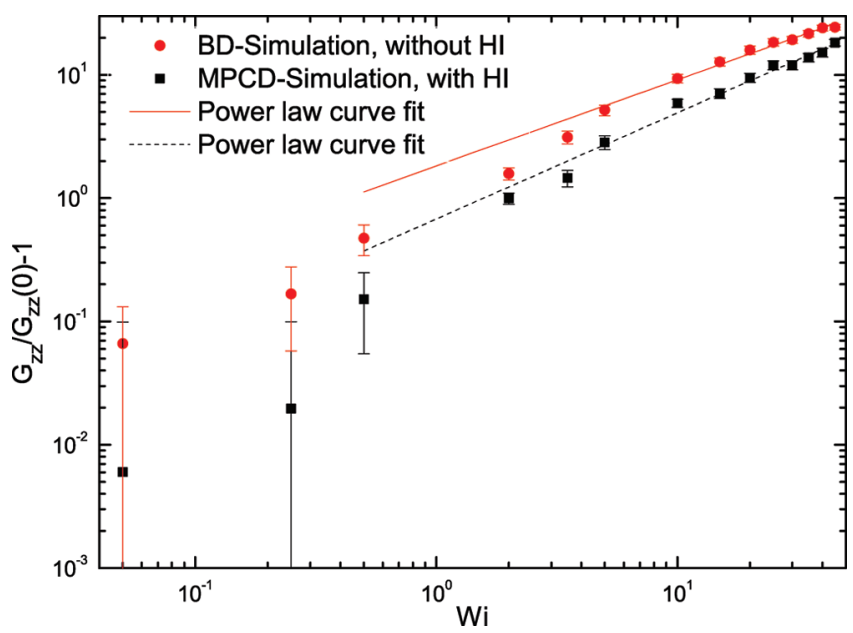

Figure 11. Same as Figure 10 but for the normalized component $G_{z z}$ of the average gyration tensor.

given by

$$
\sigma_{r}{ }^{2}=\Delta t \frac{k_{\mathrm{B}} T}{m \xi}\left(2-\frac{3-4 \mathrm{e}^{-\xi \Delta t}+\mathrm{e}^{-2 \xi \Delta t}}{\xi \Delta t}\right)
$$

Figure 10 shows the diagonal components of the gyration tensor for simulations both with and without hydrodynamic interactions. It can be seen that in the latter case the dendrimer is considerably more elongated in both the shear and the vorticity directions. This is due to the absence of coupling with the solvent particles, which creates a certain backflow driving the outer monomers toward the dendrimer's center of mass. However, this is not the case in the gradient direction, where both simulations yield almost identical results. Thus, it appears that the results do not differ qualitatively but only by a constant offset. On closer examination, however, it is visible that this is not exactly the case. This is especially evident in Figure 11, since the two curves are not parallel. This finding is also supported by the value of the determined exponent $\mu=$ $0.690 \pm 0.038$, which is substantially smaller than the one found in the case with incorporated hydrodynamics. This discrepancy is caused by the upper size limit, namely the maximum extension of a dendrimer $L_{\max }=(2 G+1) r_{0}$, which forbids constant growth. Thus, without hydrodynamics, the relative growth of the dendrimer has to be slower.

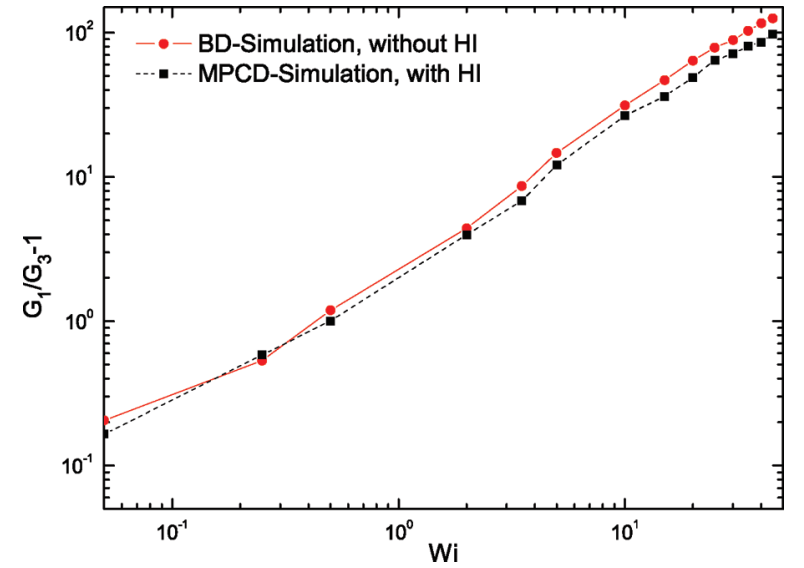

Figure 12. Same as Figure 10 but for the ratio of the largest $\left(G_{1}\right)$ and smallest $\left(G_{3}\right)$ eigenvalues of the average gyration tensor.

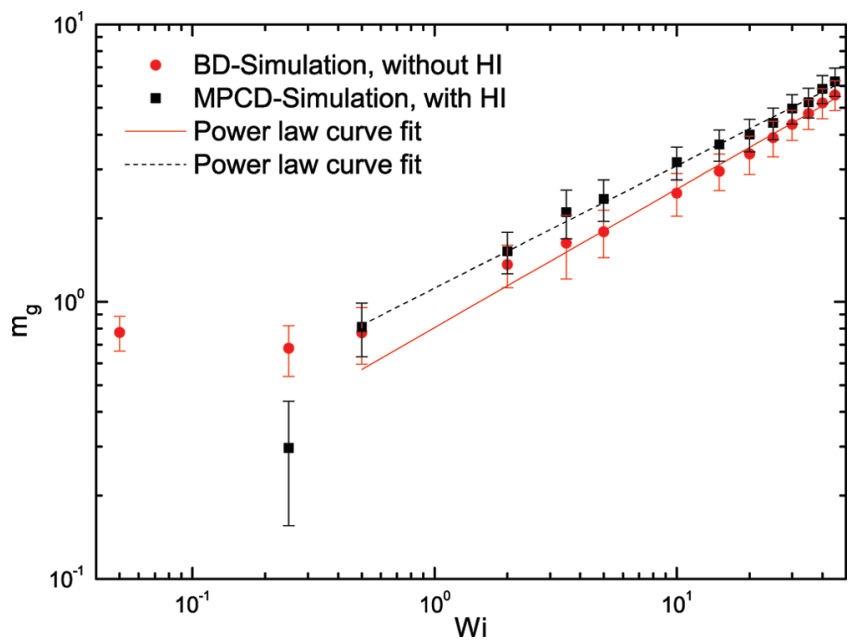

Figure 13. Same as Figure 10 but for the orientational resistance $m_{G}$.

In Figure 12 we present the asphericity $G_{3} / G_{1}-1$ of a G4-dendrimer against the Weissenberg number $W i$. Not unexpectedly, this quantity is larger in the absence of hydrodynamics but only to a small extent.

The orientational resistance $m_{G}$ however (Figure 13) shows more marked differences, depending on the lack of or presence of hydrodynamic interactions. First, unlike the results from the MPCD simulation, the values from the Brownian dynamics simulation approach a plateau for small shear rates, as expected. Second, the dendrimer exhibits a weaker orientational resistance in the absence of hydrodynamics, while, on the other hand, the slope of the fitted curve $\mu=0.500 \pm 0.020$ is somewhat larger than in the case of an explicitly modeled solvent. This behavior becomes immediately clear when Figure 8 is considered: the dendrimer does not counteract on the solvent, and thus it can be more easily brought to alignment along the shear direction.

C. Charged Dendrimers. Since the pioneering work of Welch and Muthukumar, ${ }^{44}$ charged dendrimers have attracted a lot of attention because charge can be regulated by $\mathrm{pH}$ and salinity and offers a possibility to control the dendrimer's shape externally; for recent work, see ref 45 and citations therein. Charged dendrimers under shear flow are of a special practical interest, since the possibility to induce conformational changes by the addition of salt makes them suitable candidate carrier molecules for drug delivery in the bloodstream. In this work, G4-dendrimers with monovalently $\left(Z_{\mathrm{m}}=1\right)$ and divalently $\left(Z_{\mathrm{m}}=2\right)$ charged end groups 
Table 1. Radii of Gyration for Neutral and Terminally Charged G4-Dendrimers, Depending on the Valency of Monomers, $Z_{\mathrm{m}}$, and Counterions, $Z_{\mathrm{c}}$

\begin{tabular}{lc}
\hline$\left(Z_{\mathrm{m}}, Z_{\mathrm{c}}\right)$ & $R_{\mathrm{g}} / \sigma$ \\
\hline$(0,0)$ & $2.78 \pm 0.03$ \\
$(1,1)$ & $3.04 \pm 0.04$ \\
$(2,1)$ & $3.18 \pm 0.04$
\end{tabular}
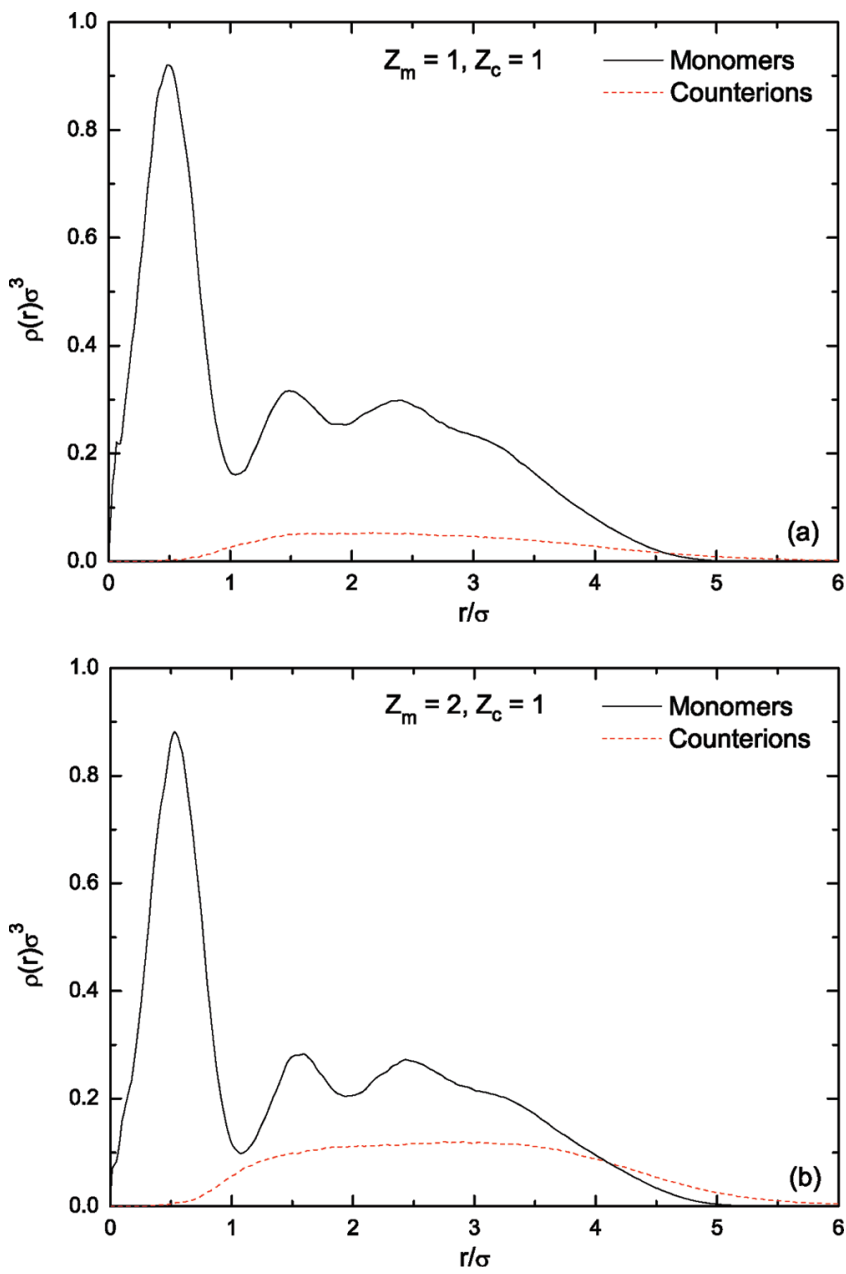

Figure 14. Equilibrium radial density distributions of two G4-dendrimers with $f=3$ and $P=0$ and charged terminal groups: (a) monovalent end groups; (b) divalent end groups.

have been studied in shear flow. Charge neutrality is restored by addition of monovalent counterions $\left(Z_{\mathrm{c}}=1\right)$. The initial configurations was equilibrated over times long enough for the counterions to diffuse into the core of the dendrimer and to reach a steady state for an in- and out-flux.

Table 1 shows the measured radii of gyration $R_{\mathrm{g}}$ for neutral and charged dendrimers. In agreement with recent work, ${ }^{45}$ we find that the size of a dendrimer increases by charging it. This swelling of the dendrimers can be understood as an osmotic effect, stemming from the electrostatic attraction between the outermost dendrimer layer and the counterions, which leads to a significant transport of counterions into the deeper regions of the dendrimer. Concomitantly, the dendrimer swells to create the necessary space for the counterions, in full analogy with the case of charged star polymers and brushes. ${ }^{46}$ This behavior is even more pronounced in the case $Z_{m}=2$, since more counterions are required to restore charge neutrality. These effects are clearly visible in Figure 14, where the radial density profiles of the monomers and counterions, both measured with respect to

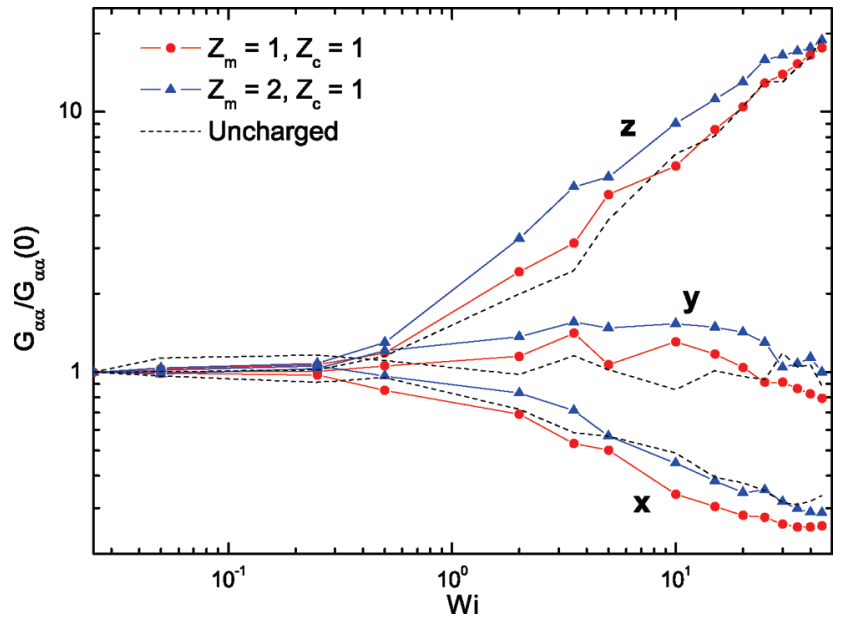

Figure 15. Normalized diagonal components $G_{\alpha \alpha}$ of the average gyration tensor as a function of $W i$, comparing between neutral and charged dendrimers.

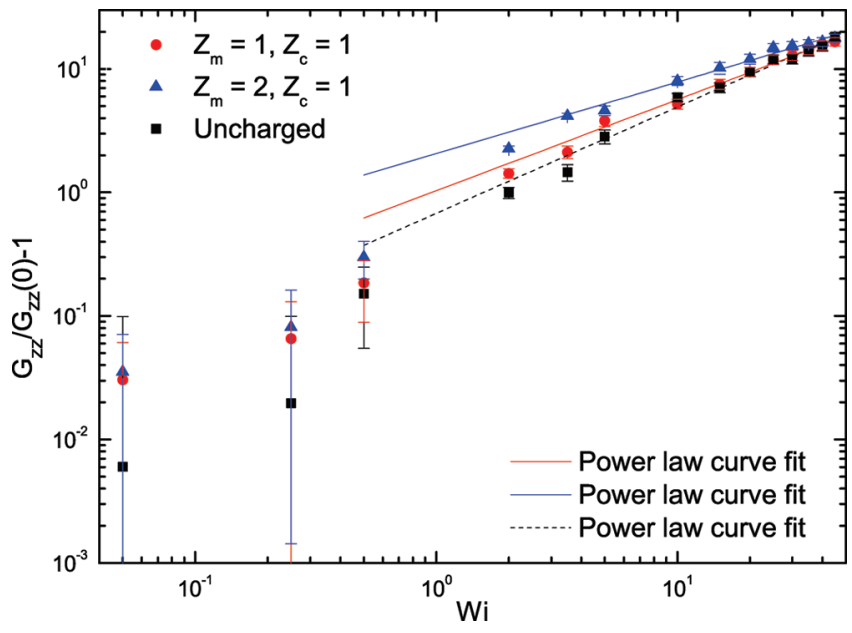

Figure 16. Same as Figure 15 but for the normalized component $G_{z z}$ of the average gyration tensor.

the dendrimer's center of mass, are shown. It is evident that the presence of charge results in a much more structured density profile because, to a certain degree, the counterions can fit in-between the individual monomer layers. Also, this effect is slightly more pronounced in the divalent case, since twice as many counterions are present.

The normalized components $G_{\alpha \alpha}$ of the average gyration tensor against the Weissenberg number $W i$, for both $Z_{m}=1$ and $Z_{m}=2$, are shown in Figure 15. It can be seen that the presence of charge leads to a slightly different behavior compared to the neutral case. Here, the deformation of the charged dendrimers is notably more pronounced in both the shear and vorticity directions. Furthermore, it is apparent that this behavior is closely related to the valency of the charged monomer layer. At larger shear rates, however, these differences disappear and the relative growths coincide with one another. These effects are even more apparent in Figure 16, where the relative extension in shear direction is plotted. The quantitative results from the curve fitting are $G_{z z} / G_{z z}(0) \approx W i^{0.74}+1$ for $Z_{m}=1$ and $G_{z z} / G_{z z}(0)=2 W i^{0.58}$ +1 in the case of $Z_{m}=2$. It is thereby easier to deform a G4-dendrimer with divalently charged end groups than its neutral or monovalent counterparts. Here, the smaller exponent is again due to the limiting length of a completely extended dendrimer. This effect becomes more clear when 


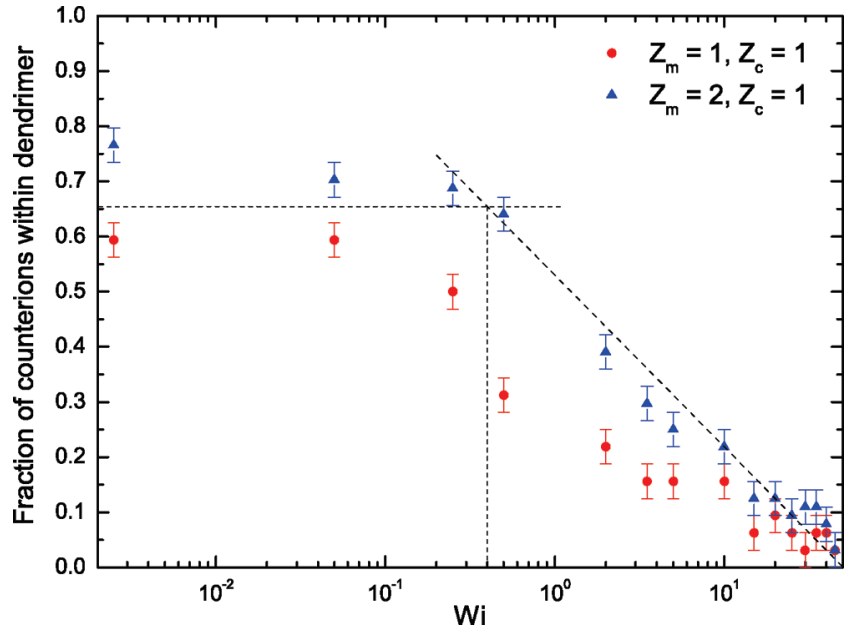

Figure 17. Fraction of counterions within the charged dendrimer as a function of the Weissenberg number $W i$. The dashed lines are guides to the eye.

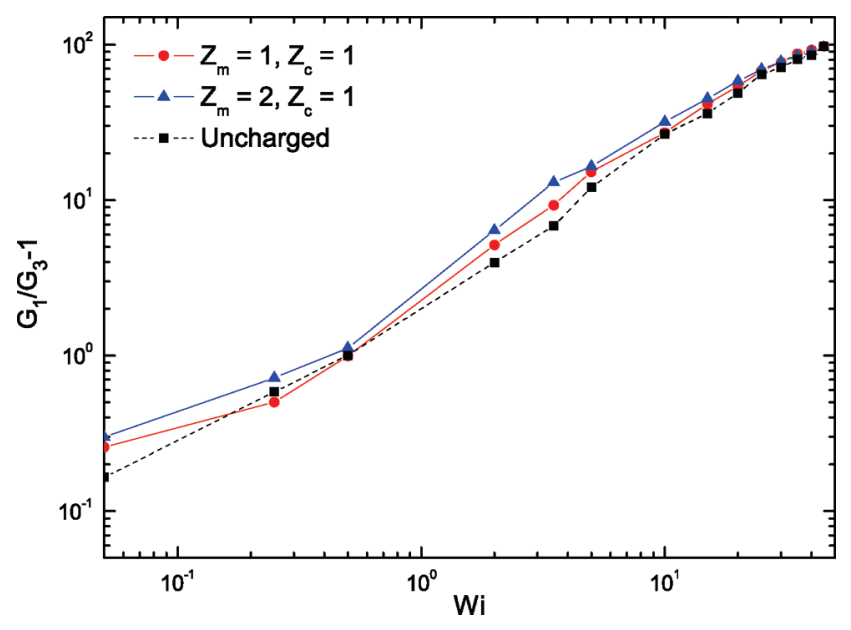

Figure 18. Same as Figure 15 but for the ratio of the largest $\left(G_{1}\right)$ and smallest $\left(G_{3}\right)$ eigenvalues of the average gyration tensor.

the fraction of counterions within the dendrimer is considered (see Figure 17). The number of trapped counterions in equilibrium or in low shear rates is considerably higher for the case of divalent end groups, a manifestation of the stronger Coulomb attraction between monomers and counterions. ${ }^{45}$ This portion then remains constant until the shear rate exceeds a critical value, which seems to be slightly higher in the case of $Z_{\mathrm{m}}=2$ (see also below). With increasing shear rates, the counterions are rinsed out of the dendrimer, since the gain in speed they acquire through collisions with the applied sheared solvent outweighs the restoring Coulomb forces. These free counterions then attract the outer shell of the dendrimer, resulting in a slightly bigger deformation in both the shear and vorticity directions. At a shear rate corresponding to $W i \gtrsim 30$, the solvent flow is sufficiently strong to fully deplete the charged dendrimers from the counterions in their interior, irrespective of the valency of the terminal monomers. Figure 18 shows the asphericity of neutral, monovalent, and divalent G4-dendrimers, and small differences between them can be observed. The reason for this lies in the loose bonding of the counterions, which first become detached from the dendrimer and flow freely along the imposed velocity field profile. These newly released, free counterions form an asymmetric cloud in the exterior of the dendrimer and attract the oppositely charged external layer

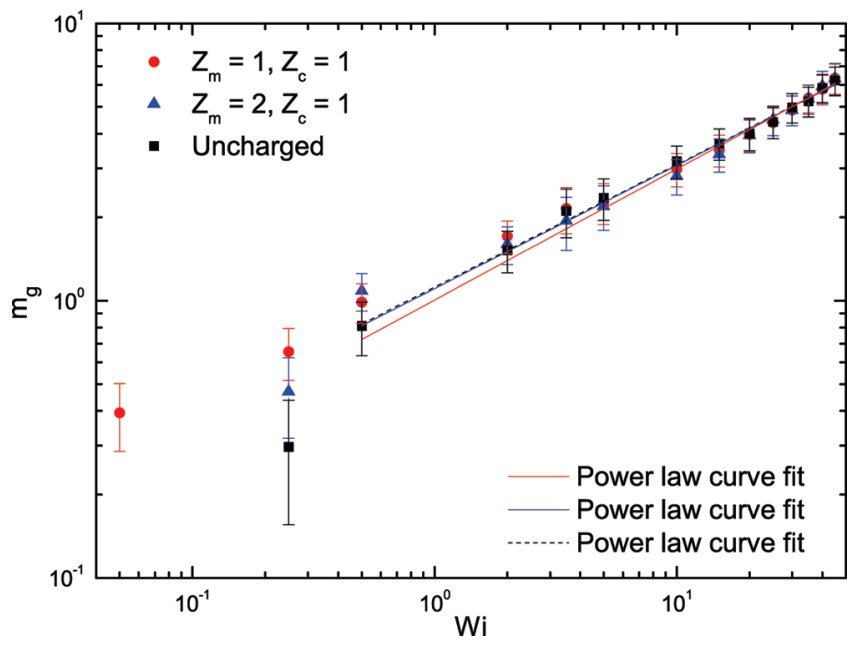

Figure 19. Same as Figure 15 but for the orientational resistance $m_{G}$.

of monomers, increasing the dendrimer's anisotropy. The orientational resistance of charged dendrimers is presented in Figure 19, and surprisingly, no remarkable differences in comparison with neutral dendrimers are visible. Moreover, the values of the exponents do not differ notably, with $\mu=$ $0.444 \pm 0.021$ for $Z_{\mathrm{m}}=1$ and $\mu=0.472 \pm 0.024$ in the case of $Z_{\mathrm{m}}=2$. Though this seems surprising at first, since one would expect a higher resistance for a charged dendrimer, this behavior can again be explained by considering Figure 17; at high shear rates almost no counterions are present within the dendrimer, which exert additional forces that try to align the polymer with the flow, while the intramonomer repulsions are dominated by the strong external flow. As a result, the polymer behaves like its neutral counterpart.

A theoretical description of the counterion distribution at equilibrium and low shear rates as well as of the physics behind the escape of the counterions from the dendrimer's interior can be reached within Poisson-Boltzmann (PB) theory. To this goal, we have solved the PB nonlinear differential equation in a spherical shell of radius $L_{\mathrm{B}}$ :

$$
\nabla^{2} \Phi(r)=-4 \pi \lambda_{\mathrm{B}}\left[Z_{\mathrm{m}} n_{\mathrm{m}}(r)+Z_{\mathrm{c}} n_{\mathrm{c}}^{\infty} \mathrm{e}^{-\beta Z_{\mathrm{c}} \Phi(r)}\right]
$$

where $n_{\mathrm{m}}(r)$ denotes the number density profile of the positive monomers, $n_{\mathrm{c}}^{\infty}$ the average number density of the negative counterions, $\beta=T^{1}$, and $L_{\mathrm{B}}$ is determined through the relation $4 \pi L_{\mathrm{B}}{ }^{3} / 3=V$. Moreover, the boundary conditions $\nabla \Phi(0)=0$ and $\nabla \Phi\left(L_{\mathrm{B}}\right)=0$ have been applied, where the former stems from symmetry reasons and the latter is an expression of the overall charge neutrality. We emphasize that the quantity $n_{\mathrm{m}}(r)$ is not calculated within the present, Poisson-Boltzmann approach but it is rather an input to it, taken directly from the simulation results.

The nonlinear Poisson-Boltzmann differential equation (15) presents only an approximation, since it does not take any spatial accessibility into account and thus disregards excluded volume effects, and even as far as the charge response is concerned, it is at the level of a mean-field approximation. Nonetheless, the solutions of this equation are surprisingly accurate. Figure 20 shows the resulting counterion density, and a good agreement between the simulation and the approximation can be observed. This holds especially true in the case of $Z_{\mathrm{m}}=2$, since, with increasing valency, the Coulomb forces play a bigger role and more counterions are trapped inside the dendrimer. The fact that excluded volume effects have been disregarded is well visible, since the calculated counterion density at the dendrimer's center of 

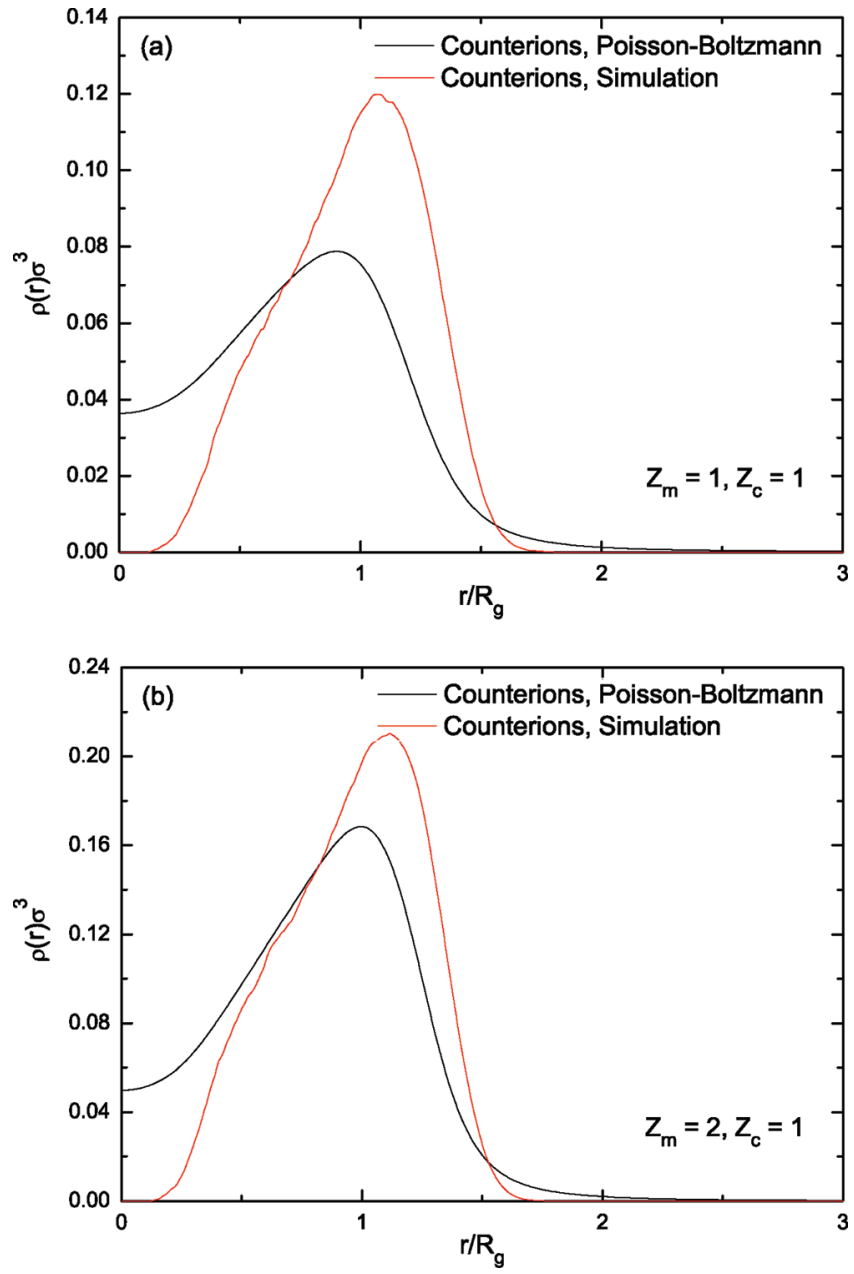

Figure 20. Radial density distribution of the counterions of the two terminally charged dendrimers considered in this work. The black curves represent the solution of the Poisson-Boltzmann equation (15), while the red curves are the simulation results. (a) Charged dendrimer with monovalent end groups. (b) Charged dendrimer with divalent end groups.

mass does not converge to zero. The resulting electrostatic potentials and energies are shown in Figure 21. Here, the relevant energy $E_{\mathrm{esc}}$, which has to be gained by a trapped counterion in order to overcome the barrier and escape from the interior of the polymer, is given by the difference between the minimum energy and the value at the edge of the system. This required energy, expressed in thermal units, reads as $E_{\mathrm{esc}} \approx 8.4$ for $Z_{\mathrm{m}}=1$ and $E_{\mathrm{esc}} \approx 8.6$ for $Z_{\mathrm{m}}=2$.

An estimate of the critical Weissenberg number $W i_{\mathrm{c}}=\tau \dot{\gamma}_{\mathrm{c}}$, which leads to the rinsing of the counterions, can be made as follows. The values above have to be compared to the local increase in kinetic energy due to the shear flow. First, the velocity in shear direction at the position $x$ is given by

$$
v_{z}(x)=2 \dot{\gamma}\left(x-\frac{L_{x}}{2}\right)
$$

Since the kinetic energy due to the Couette flow has to equal the escape energy $E_{\mathrm{esc}}$, the expression for the critical shear rate reads as

$$
W i_{\mathrm{c}}=\tau\left|\sqrt{\frac{E_{\mathrm{esc}}}{2 m}}\left(R_{\mathrm{g}}-\frac{L_{x}}{2}\right)^{-1}\right|
$$

After inserting the simulation parameters $m=5, \tau=47.2$ and the corresponding values for $R_{\mathrm{g}}$ and $E_{\mathrm{esc}}$, eq 17 yields the
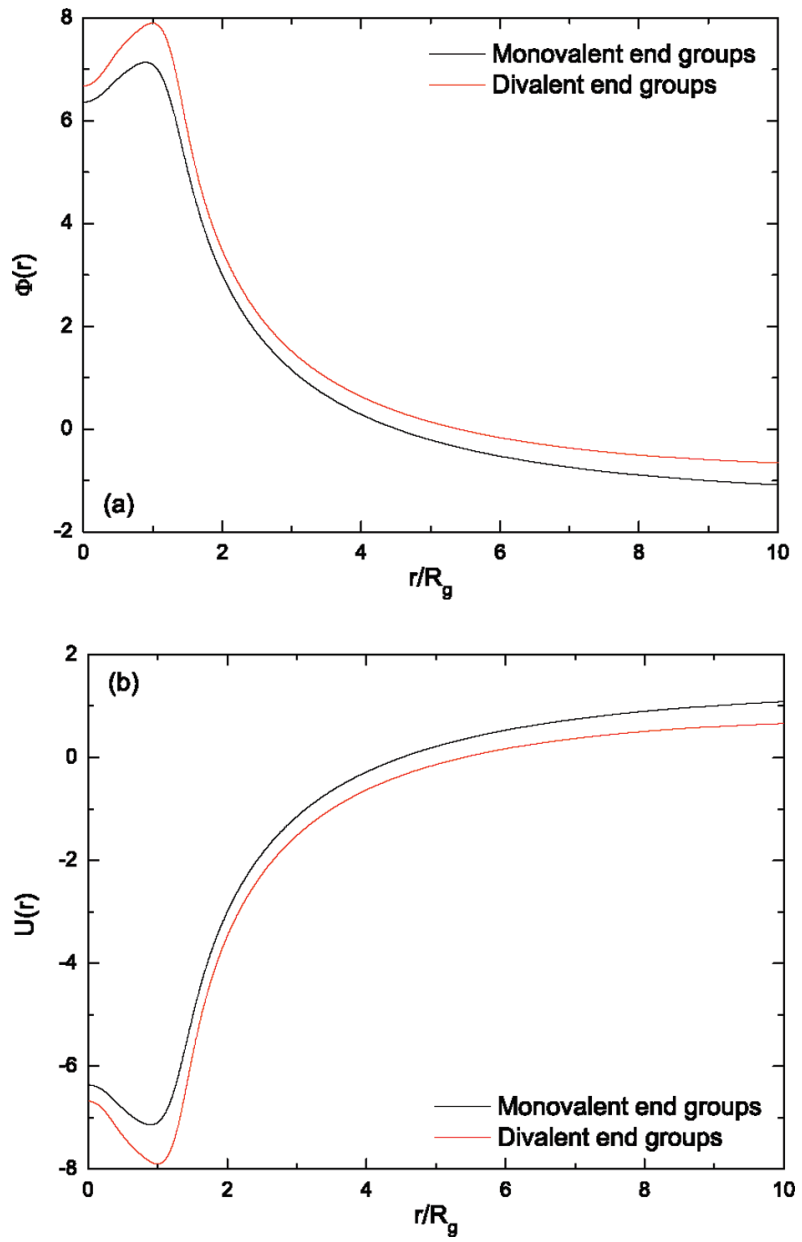

Figure 21. (a) Electrostatic potentials and (b) energies per counterion of G4-dendrimers with monovalent and divalent end groups.

critical Weissenberg numbers $W i_{\mathrm{c}} \cong 1.2$ for both monovalent and divalent dendrimers. The simulation result for this quantity can be read off from Figure 17, where it can be identified with the point of crossover from a constant value for the fraction of trapped counterions to a monotonically decreasing quantity; this occurs roughly at $W i \cong 0.5$. The overestimation from theory originates from various assumptions. Among these is, for example, the fact that the majority of counterions has been assumed to be located at $r \approx R_{\mathrm{g}}$. However, on closer inspection it is apparent that the simulated counterion cloud lies slightly further away from the dendrimer's center of mass than the calculated one. Consequently, the counterions are exposed to a stronger current, and hence smaller Weissenberg numbers are needed to release them. Nonetheless, these results reproduce the findings of the simulation quite accurately and predict the qualitative behavior of the counterions correctly.

\section{Conclusions}

We have carried out hybrid simulations at the particle-resolved level to analyze the shape and response of regularly branched polymers under shear flow. We have shown that dendrimers under shear exhibit a very rich structural and dynamical behavior, which distinctively differs from that of linear and star polymers. Moreover, we have demonstrated in this contribution that hydrodynamic interactions play an important role for the conformational properties of dendritic structures, since their strong influence on the fluid leads to an unique flow profile in their interior and vicinity. This behavior is strongly related to the 
dendrimers' many conformational degrees of freedom. A considerable screening of the externally imposed flow field has been observed in the area in which the fluid coexists with the polymer. Outside this region, the fluid adapts to the central rotation by generating a counter-rotating vortex, which leads to a substantial backfolding of the dendrimer's outer monomer layers. Hence, the incorporation of hydrodynamic interactions leads to a notably smaller elongation of the polymer. This behavior has been quantified in this work by means of Brownian dynamics simulations, in which the hydrodynamics have been omitted.

The characteristics of dendritic structures with charged end groups have been analyzed under shear conditions, and it has been shown that the majority of counterions remains inside the polymer until the shear rate exceeds a certain critical value. Beyond this rate, however, the counterions get rinsed out of the macromolecule rather quickly, and the dendrimer starts to resemble more and more its neutral counterpart. These findings have also been verified analytically, by first solving the Poisson-Boltzmann equation for the counterion number density and then calculating the necessary critical shear rates approximately. This behavior opens up an additional possibility to employ charged dendrimers as candidate carrier molecules for drug delivery, since the absorbed substances become released at a well-defined shear rate. From the fundamental point of view, the next challenge is to simulate concentrated dendrimer solutions under full hydrodynamic coupling and to develop suitable coarsegraining strategies for such molecules out of equilibrium, possibly along the lines of the recently proposed transient-force approach by Briels. ${ }^{47}$

Acknowledgment. The authors thank Adam Wysocki, Marisol Ripoll, and Dimitris Vlassopoulos for helpful discussions. A.N. acknowledges the Studienstiftung des Deutschen Volkes and the SFB-TR6, Project C3, for financial support.

\section{References and Notes}

(1) Yang, H.; Lopina, S. J. Biomater. Sci., Polym. Ed. 2003, 14, 10431056.

(2) Vlassopoulos, D.; Pakula, T.; Fytas, G.; Roovers, J.; Karatasos, K.; Hadjichristidis, N. Europhys. Lett. 1997, 39, 617-622.

(3) Smith, D. E.; Babcock, H. P.; Chu, S. Science 1999, 283, 1724-1727.

(4) Gerashchenko, S.; Steinberg, V. Phys. Rev. Lett. 2006, 96, 038304.

(5) Winkler, R. G.; Mussawisade, K.; Ripoll, M.; Gompper, G. J. Phys.: Condens. Matter 2004, 16, S3941-S3954.

(6) Ripoll, M.; Winkler, R. G.; Gompper, G. Phys. Rev. Lett. 2006, 96, 188302.

(7) Ripoll, M.; Winkler, R. G.; Gompper, G. Eur. Phys. J. E 2007, 23, 349-354.

(8) Stone, H. A. Annu. Rev. Fluid Mech. 1994, 26, 65-102.

(9) Kraus, M.; Wintz, W.; Seifert, U.; Lipowsky, R. Phys. Rev. Lett. 1996, 77, 3685-3688.
(10) Götze, I. O.; Likos, C. N. Macromolecules 2003, 36, 8189-8197.

(11) Ballauff, M.; Likos, C. N. Angew. Chem., Int. Ed. 2004, 43, 29983020.

(12) Boris, D.; Rubinstein, M. Macromolecules 1996, 29, 7251-7260.

(13) Bosko, J. T.; Todd, B. D.; Sadus, R. J. J. Chem. Phys. 2004, 121, 1091-1096.

(14) Bosko, J. T.; Todd, B. D.; Sadus, R. J. J. Chem. Phys. 2004, 121, 12050-12059.

(15) Bosko, J. T.; Todd, B. D.; Sadus, R. J. J. Chem. Phys. 2006, 124, 044910

(16) Asteriadi, A.; Sigel, R.; Vlassopoulos, D.; Meier, G.; Dorgan, J. R.; Knauss, D. M. Macromolecules 2004, 37, 1016-1022.

(17) Lyulin, A. V.; Davies, G. R.; Adolf, D. B. Macromolecules 2000, 33, 3294-3304.

(18) Lyulin, A. V.; Adolf, D. B.; Davies, G. R. Macromolecules 2001, 34, 3783-3789.

(19) Bosko, J. T.; Ravi Prakash, J. J. Chem. Phys. 2008, 128, 034902.

(20) Dalakoglou, G. K.; Karatasos, K.; Lyulin, S. V.; Lyulin, A. V. J. Chem. Phys. 2008, 129, 034901.

(21) Malevanets, A.; Kapral, R. J. Chem. Phys. 1999, 110, 8605-8613.

(22) Gompper, G.; Ihle, T.; Kroll, D. M.; Winkler, R. G. Adv. Polym. Sci. 2009, 221, 1-87.

(23) Grest, G. S.; Kremer, K.; Witten, T. A. Macromolecules 1987, 20 , 1376-1383.

(24) Lieb, E. H. Rev. Mod. Phys. 1976, 48, 553-569.

(25) Likos, C. N. Phys. Rep. 2001, 348, 267-439.

(26) Ladd, A. J. C. Mol. Phys. 1984, 53, 459-463.

(27) Evans, D. J.; Moriss, G. P. Phys. Rev. A 1984, 30, 1528-1530.

(28) Kairn, T.; Davis, P. J.; Matin, M. L.; Snook, I. K. Polymer 2004, 45, 2453-2464.

(29) de Gennes, P.; Hervet, H. J. Phys., Lett. 1983, 44, 351-360.

(30) Ihle, T.; Kroll, D. M. Phys. Rev. E 2001, 63, 020201.

(31) Lamura, A.; Gompper, G.; Ihle, T.; Kroll, D. M. Europhys. Lett. 2001, 56, 319-325.

(32) Allahyarov, E.; Gompper, G. Phys. Rev. E 2002, 66, 036702.

(33) Malevanets, A.; Kapral, R. J. Chem. Phys. 2000, 112, 7260-7269.

(34) Padding, J. T.; Wysocki, A.; Lowen, H.; Louis, A. A. J. Phys.: Condens. Matter 2005, 17, S3393-S3399.

(35) Malevanets, A.; Yeomans, J. M. Europhys. Lett. 2000, 52, 231-237.

(36) Mussawisade, K.; Ripoll, M.; Winkler, R. G.; Gompper, G. J. Chem. Phys. 2005, 123, 144905.

(37) Ripoll, M.; Mussawisade, K.; Winkler, R. G.; Gompper, G. Europhys. Lett. 2004, 68, 106-112.

(38) Ewald, P. P. Ann. Phys. 1921, 369, 253-287.

(39) Allen, M. P.; Tildesley, D. J. Computer Simulation of Liquids; Oxford University Press: New York, 1989.

(40) Doi, M.; Edwards, S. F. The Theory of Polymer Dynamics; Clarendon Press: London, 1999.

(41) Link, A.; Springer, J. Macromolecules 1993, 26, 464-471.

(42) Aust, C.; Kroger, M.; Hess, S. Macromolecules 1999, 32, 56605672.

(43) Teixeira, R. E.; Babcock, H. P.; Shaqfeh, E. S. G.; Chu, S. Macromolecules 2005, 38, 581-592.

(44) Welch, P.; Muthukumar, M. Macromolecules 2000, 33, 6159-6167.

(45) Blaak, R.; Lehmann, S.; Likos, C. N. Macromolecules 2008, 41, $4452-4458$

(46) Jusufi, A.; Likos, C. N. Rev. Mod. Phys. 2009, 81, 1753-1772.

(47) Briels, W. J. Soft Matter 2009, 5, 4401-4411. 